\section{International Scientific Journal Theoretical \& Applied Science}

p-ISSN: 2308-4944 (print) e-ISSN: 2409-0085 (online)

Year: $2016 \quad$ Issue: $7 \quad$ Volume: 39

Published: $30.07 .2016 \quad$ http://T-Science.org

SECTION 20. Medicine.
Olena Svietlova

Candidate of biological sciences, senior lecturer Cherkasy National University named after Bohdan Khmelnytsky svetlova_2004@vu.cdu.edu.ua

Oksana Suprunenko Candidate of technical sciences, associate professor Cherkasy National University named after Bohdan Khmelnytsky ra-oks@mail.ru

Alevtyna Rybalko Candidate of biological sciences, teacher Cherkasy National University named after Bohdan Khmelnytsky rybalko@vu.cdu.edu.ua

Stanislav Kovalenko Doctor of biological sciences, Professor, head of the department

Cherkasy National University named after Bohdan Khmelnytsky

kovstas@cdu.edu.ua

\title{
MODELS MORBIDITY A PUPILS OF MIDDLE SCHOOL AGE
}

Abstract: The article presents the researches of the dynamics of morbidity of children of school age in the central region of Ukraine, which were conducted to predict the diseases of pupils in the planning of health improvement and preventive measures to reduce the leading disease at school age. Were constructed functional and adaptive model of morbidity children middle school age. Were presented simulation results of forecast indicators by disease classes, the structure of morbidity modelling system of pupils, analyzed the dynamics of morbidity.

Key words: child morbidity, pathological affection of model child morbidity, prediction, autoregression model.

Language: Russian

Citation: Svietlova O, Suprunenko O, Rybalko A, Kovalenko S (2016) MODELS MORBIDITY A PUPILS OF MIDDLE SCHOOL AGE. ISJ Theoretical \& Applied Science, 07 (39): 75-84.

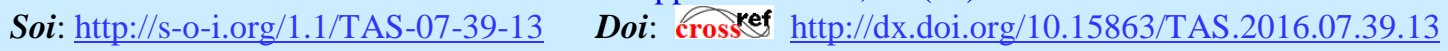

\section{МОДЕЛИ ЗАБОЛЕВАЕМОСТИ ДЕТЕЙ СРЕДНЕГО ШКОЛЬНОГО ВОЗРАСТА}

Аннотация: В статье представлены исследования динамики заболеваемости детей школьного возраста центрального региона Украины, которые проводились для прогнозирования заболеваний школьников при планировании оздоровительно-профилактических мероприятий с целью снижения ведущей патологии в школьном возрасте. Построены функииональная и адаптивная модели заболеваемости детей среднего школьного возраста. Представлены результаты моделирования, прогнознье показатели по классам болезней, структура системы моделирования заболеваемости икольников, проанализирована динамика заболеваемости.

Ключевые слова: заболеваемость детей, патологическая пораженность, модель заболеваемости детей, прогнозирование, модель авторегрессии.

Введение. В последние десятилетия наблюдается существенное увеличение заболеваемости населения, что обусловлено экологической ситуацией и рядом других факторов. Кроме влияния экологических факторов, здоровье человека формируется под действием целого комплекса медицинских, экономических и социальных факторов. Эти факторы в последние годы в Украине только обостряются. И наиболее адекватным ответом на изменение этих факторов является активное изучение влияния наиболее существенных факторов на здоровье населения, а также обоснованное и целенаправленное планирование и осуществление мероприятий по профилактике заболеваний населения, и особенно здоровья 
детей, что в перспективе окажет существенное влияние на здоровье большей части населения Украины.

\section{1. Анализ литературных источников, постановка проблемы}

Современные исследования учёных [1-5] убедительно подтверждают ухудшение состояния здоровья населения Украины и соседних с ней стран Европейского Союза. Так в $6-8$ раз увеличилось число онкологических заболеваний; в 1,5 - 6 раз возросли показатели заболеваемости по различным неонкологическим классам болезней [1-4].

Одним из самых значительных на сегодня в Украине является экономический кризис, который еще больше углубляется военным конфликтом. В свою очередь финансовая нестабильность приводит к низкому уровню финансирования сферы охраны здоровья и медицинского образования, что снижает эффективность и качество медицинского обслуживания. Этот фактор также является одной из причин возрастания заболеваемости населения [6-7]. Усугубляют сложившуюся ситуацию и устаревшие представления, которые относятся к общественному здоровью, консервативное отношение специалистов к новейшим технологиям в медицине и образовательной сфере, отсутствие связей между научными исследованиями и практикой [7].

Приостановление значительных негативных процессов в состоянии здоровья населения в стране можно ожидать лишь постоянно контролируя уровень здоровья всех его слоев. Но в данное время в ЦентральноВосточноевропейском регионе, к которому относится и Украина, проводится недостаточно исследований, которые направлены на изучение состояния здоровья населения [8-9]. Так, в комментариях [8] особенно подчеркивается необходимость проведения глобальных исследований в сфере охраны здоровья, которые будут способствовать осуществлению профилактических мероприятий, направленных на оздоровление населения и снижения уровня заболеваемости.

Поскольку известно [8-9], что залогом успешного планирования и осуществления оздоровительных программ являются данные о состоянии здоровья населения, ретроспективный анализ динамики заболеваемости с последующим прогнозированием возможного уровня отдельных классов заболеваний является актуальным предметом исследования.

Состояние здоровья населения зависит от потенциала здоровья людей, заложенного в детском возрасте [8], потому особенно актуальными являются исследования динамики заболеваемости детей школьного возраста, как в общем, так и по отдельным классам заболеваний.

\section{2. Цель и задачи исследования}

Целью данного исследования является моделирование и изучение на моделях заболеваемости детей школьного возраста центрального региона Украины за последние тридцать лет, а также прогнозирование дальнейшей динамики патологической пораженности школьников.

Для реализации поставленной цели решались следующие задачи:

1. Провести ретроспективный анализ состояния здоровья детей школьного возраста (центральный регион Украины) за последние тридцать лет.

2. Построить модели заболеваемости школьников по данным 1986-2015 годов.

3. Проанализировать на моделях динамику заболеваемости школьников на протяжение вышеуказанного периода.

4. Изучить характер изменений патологической поражённости школьников по отдельным группам и формам заболеваний.

5. Оценить модели заболеваемости школьников и спрогнозировать дальнейшую динамику изменений заболеваемости детей школьного возраста.

\section{3. Методы исследования здоровья детей} школьного возраста

$\begin{array}{ccc}\text { Общая } & \text { организация } & \text { исследования } \\ \text { здоровья } & \text { школьников. } & \text { Исследования }\end{array}$
предусматривали использование эпидемиологического метода, теоретического анализа и обобщения, метода математического прогнозирования временных рядов.

В центральном регионе Украины, на примере г. Черкассы, исследовали состояние здоровья школьников 6 - 16 лет. Динамику заболеваемости учеников изучали по данным официальной статистики за период 1986-2015 годов. Наблюдениями было охвачено 534326 детей школьного возраста.

За период ретроспективного изучения состояния здоровья школьников медицинские обзоры проводились в соответствии с действующими государственными нормативноправовыми документами, в том числе: Постановление КМУ за № 1318 об утверждении «Порядка осуществления медицинского обслуживания учеников общеобразовательных учебных заведений» [10]; Приказ МO3 Украины за № 682 «Об усовершенствовании медицинского обслуживания учеников общеобразовательных учебных заведений» [11]. 


\begin{tabular}{|c|c|c|c|c|c|c|}
\hline Impact Factor: & $\begin{array}{l}\text { ISRA (India) } \\
\text { ISI (Dubai, UAE } \\
\text { GIF (Australia) } \\
\text { JIF }\end{array}$ & $\begin{array}{l}=1.344 \\
=0.829 \\
=0.564 \\
=1.500\end{array}$ & $\begin{array}{l}\text { SIS (USA) } \\
\text { PИНЦ (Russia) } \\
\text { ESJI (KZ) } \\
\text { SJIF (Morocco) }\end{array}$ & $\begin{array}{l}=0.912 \\
=0.234 \\
=1.042 \\
=2.031\end{array}$ & $\begin{array}{l}\text { ICV (Poland) } \\
\text { PIF (India) } \\
\text { IBI (India) }\end{array}$ & $\begin{array}{l}=6.630 \\
=1.940 \\
=4.260\end{array}$ \\
\hline
\end{tabular}

Математические методы анализа заболеваемости. Общий коэффициент заболеваемости (общая патологическая пораженность) и коэффициенты заболеваемости по отдельным нозологиями определялись по формуле:

$$
P=\frac{n}{N} \times 1000(\%)
$$

где $P$ - коэффициент общей патологической пораженности, или отдельной нозологии (\%o), $n-$ количество выявленной патологии, $N$ - общее число осмотренных лиц.

Погрешность среднего значения рассчитывалась по формуле:

$$
m=\sqrt{\frac{P \times\left(1000^{-P}\right)}{N}}(\%)
$$

где $P$ - коэффициент общей патологической пораженности или отдельной нозологии (\%o), $N-$ общее число осмотренных лиц.

Оценка динамических изменений коэффициентов заболеваемости по отдельным группам и формам болезней осуществляли с помощью характеристики динамических рядов, анализ которых состоял из нескольких этапов:

1) определялся абсолютный прирост коэффициенту по формуле:

$$
\text { Anp }=P_{i}-P_{0} \quad(\% o)
$$

где $A n p$ - абсолютный прирост (\%o), $P_{i}-$ уровень текущего периода $(\%), P_{O}-$ уровень базисного периода $(\%)$;

2) вычислялся темп прироста коэффициента по формуле:

$$
\operatorname{Tn} p=\frac{P_{i}-P_{0}}{P_{0}} \times 100(\%)
$$

где $T n p$ - темп прироста $(\%), P_{i}-$ уровень текущего периода $(\%), P_{O}-$ уровень базисного периода $(\%)$;

3) вычислялся темп роста коэффициента по формуле:

$$
T p=\frac{P_{i}}{P_{0}} \times 100(\%)
$$

где $T p$ - темп роста $(\%), \boldsymbol{P}_{i}$ - уровень текущего периода $(\%), P_{o}-$ уровень базисного периода (\%o).

Отличия между показателями оценивались по критерию Стьюдента $(t)$ :

$$
t=\frac{\left|P_{1}-P_{2}\right|}{\sqrt{m_{1}^{2}+m_{2}^{2}}}
$$

где $P$ - коэффициенты общей патологической пораженности или отдельной нозологии (\%o), $m-$ погрешности их средних значений (\%o).

Структура заболеваемости по группам и формам болезней изучалась по общепринятым методикам санитарной статистики, по формулам 7, 8:

$$
P=\frac{n}{N} \times 100(\%)
$$

где $P$ - процент нозологии $(\%), n-$ количество выявленных заболеваний определенной нозологической формы, $N$ - общее число заболеваний.

$$
m=\sqrt{\frac{P \times\left(100^{-} P\right)}{N}}(\%)
$$

где $P$ - коэффициенты общей патологической пораженности или отдельной нозологии (\%), $N$ общее число заболеваний.

Согласно полученным данным патологической пораженности школьников, строились графики, которые позволили проследить характер и динамические изменения ряда заболеваний. Для того, чтобы уменьшить влияние на динамический ряд случайных факторов и для выявления основной тенденции развития явления, проводилось выравнивание динамического ряда. Для этого использовалась методика «сглаживания», суть которой заключается в том, что каждый показатель заменяется средним значением показателя и окружающих его элементов ряда.

Методика построения моделей заболеваемости. Полученные графики дали возможность определить типы зависимостей, и в результате выделить восходящие, нисходящие и восходящиенисходящие / нисходящие-восходящие функциональные модели для прогнозирования на кратковременную перспективу уровня патологической пораженности школьников. Восходящие и нисходящие модели описывались с помощью линейной функции по формуле:

$$
y=a+b t
$$

где $в-\%$ нозологии; $a, b$ - коэффициенты модели; $t-$ время (в годах).

Для описания функциональных моделей, которые имеют выраженный восходящийнисходящий (или нисходящий-восходящий) характер, использовались модели полиномов 2-го порядка:

$$
y=a+b t+c t^{2}
$$

где $в-\%$ нозологии; $a, b, c-$ коэффициенты модели; $t$ - время (в годах).

При анализе графиков заболеваемости школьников среднего школьного возраста были определены характер и динамика изменений показателей патологической поражений школьников, что позволило сформировать адаптивную модель заболеваемости школьников среднего школьного возраста. Для построения модели использовалась модель авторегрессии или стохастическое разностное уравнение: 


\begin{tabular}{|c|c|c|c|c|c|c|}
\hline Impact Factor: & $\begin{array}{l}\text { ISRA (India) } \\
\text { ISI (Dubai, UAB } \\
\text { GIF (Australia) } \\
\text { JIF }\end{array}$ & $\begin{array}{r}=1.344 \\
=0.829 \\
=0.564 \\
=1.500\end{array}$ & $\begin{array}{l}\text { SIS (USA) } \\
\text { PИHЦ (Russia) } \\
\text { ESJI (KZ) } \\
\text { SJIF (Morocco) }\end{array}$ & $\begin{array}{l}=0.912 \\
=0.234 \\
=1.042 \\
=2.031\end{array}$ & $\begin{array}{l}\text { ICV (Poland) } \\
\text { PIF (India) } \\
\text { IBI (India) }\end{array}$ & $\begin{array}{l}=6.630 \\
=1.940 \\
=4.260\end{array}$ \\
\hline
\end{tabular}

$$
x_{i}=a_{1} x_{i-1}+\varepsilon_{i}
$$

Выбранная модель представляет узкий класс гаусовских марковских случайных процессов с экспоненциальной корреляционной функцией [12]. Марковским процесс является тогда, когда последующее значение определяется только предшествующим, без учета предыдущих значений числового ряда. Поскольку полученные значения числового ряда заболеваемости школьников не являются стационарными для их описания требуется модель, которая учитывает большее количество статистических факторов. Классическая модель авторегрессии может быть преобразована в авторегрессионную модель более высоких порядков, в этом случае она будет описывать уже не марковские случайные процессы. В качестве основы для моделирования заболеваемости учеников среднего школьного возраста была выбрана авторегрессионная модель второго порядка [13]:

$$
M_{i}=a_{1 i} M_{i-1}+a_{2 i} M_{i-2}+\varepsilon_{i}
$$

где $a_{1 i}, a_{2 i}$ - коэффициенты авторегрессии, $\varepsilon_{i}$ некоррелированная случайная компонента, отображающая ошибки измерений и погрешности модели. Адаптивная модель заболеваемости школьников строилась с учетом сглаженных значений $\boldsymbol{P}_{i}$, что позволило снизить влияние на динамический ряд случайных факторов. Коэффициент $a_{1 i}$ фактически представляет собой коэффициент корреляции между тремя значениями $\left\{\boldsymbol{P}_{i-2}, \boldsymbol{P}_{i-1}, \boldsymbol{P}_{i}\right\} \quad$ и $\quad$ тремя соответствующими сглаженными значениями. Коэффициент $a_{2 i}$ вычислялся из условий ограничения допустимых значений $a_{1}$ и $a_{2}$ в корреляционной функции $[13,14]$. Предложенная адаптивная модель предусматривает расчет коэффициентов авторегрессии на каждом шаге итерации, начиная з третьего элемента числового ряда. При таком подходе на последующих 12-15 значениях числового ряда ошибка модели затухает, что позволяет проводить краткосрочное прогнозирование последующих значений с оценкой погрешности до $12 \%$.

Для первичной обработки информации и получения промежуточных расчетов использовали пакет Excel MS Office, основная часть математической обработки была выполнена с использованием стандартного статистического пакета STATISTICA 5.5.

\section{4. Результаты исследования состояния здоровья школьников}

Анализ результатов профилактических медицинских обследований школьников центрального региона Украины позволил провести оценку динамики состояния здоровья учеников 6-16 лет за последние тридцать лет, указывая на существенное повышение заболеваемости школьников с 385,9 \% $\pm 4,07 \%$ в 1986 году до 810,4\% \pm 2,47\%о в 2015 году ( $\mathrm{p}<0,001)$. Однако, на протяжении последнего десятилетия, начиная с 2004 - 2005 годов, отмечается приостановка роста уровня патологической пораженности школьников (рис. 1).

Проведенная оценка динамических изменений заболеваемости по отдельным группам и формами болезней показала, что на протяжении $1986-2015$ годов уровень патологической пораженности детей и подростков повышался. Эти изменения происходили за счет роста болезней системы кровообращения в 3,5 раза, болезней глаза и придаточного аппарата в 1,9 раза, болезней и функциональных нарушений костно-мышечной системы в 2,8 раза, болезней эндокринной системы в 3,3 раза, расстройства питания и нарушений обмена веществ в 1,3 раза, болезней системы дыхания в 1,8 раза (табл. 1).

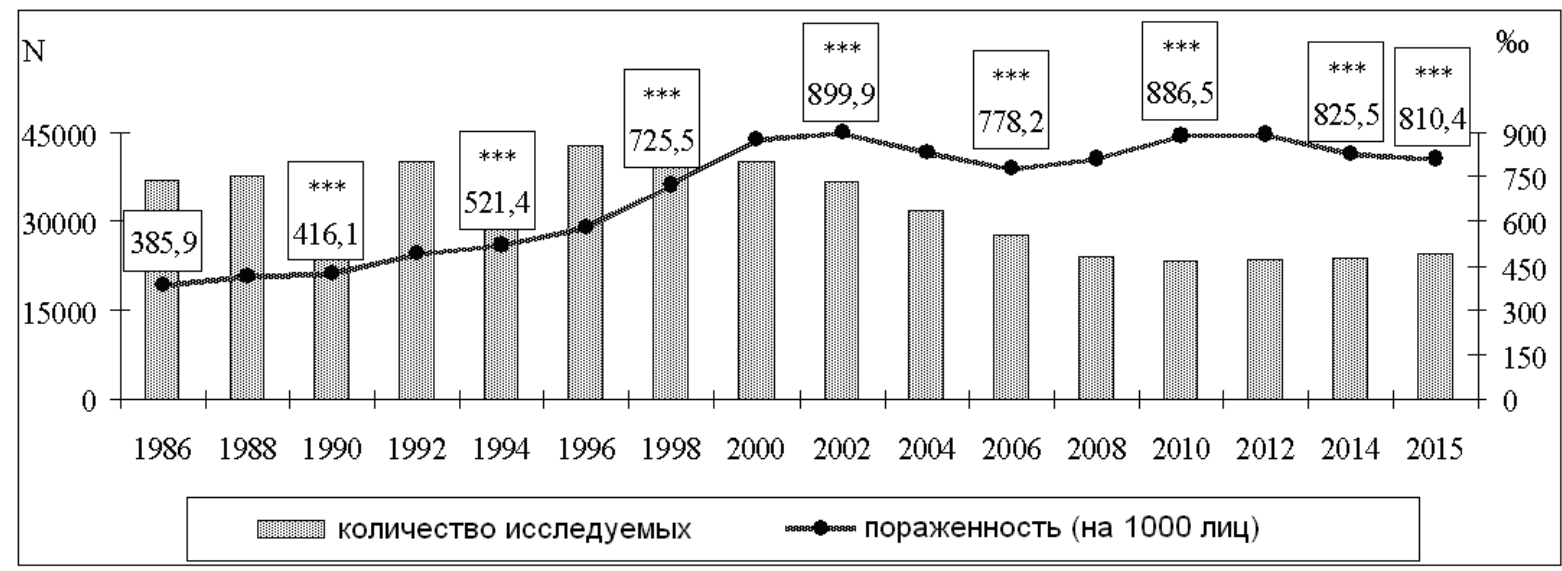

Рисунок 1 - Динамика патологической пораженности школьников 6 - 16 лет города Черкассы (интенсивные показатели \%о): *** - p < 0,001 при парном сравнении.

ISPC Results and Perspectives,

Harrisburg, USA 


\begin{tabular}{|c|c|c|c|c|c|c|}
\hline Impact Factor: & $\begin{array}{l}\text { ISRA (India) } \\
\text { ISI (Dubai, UAB } \\
\text { GIF (Australia) } \\
\text { JIF }\end{array}$ & $\begin{array}{r}=1.344 \\
=0.829 \\
=0.564 \\
=1.500\end{array}$ & $\begin{array}{l}\text { SIS (USA) } \\
\text { PИHЦ (Russia) } \\
\text { ESJI (KZ) } \\
\text { SJIF (Morocco) }\end{array}$ & $\begin{array}{l}=0.912 \\
=0.234 \\
=1.042 \\
=2.031\end{array}$ & $\begin{array}{l}\text { ICV (Poland) } \\
\text { PIF (India) } \\
\text { IBI (India) }\end{array}$ & $\begin{array}{l}=6.630 \\
=1.940 \\
=4.260\end{array}$ \\
\hline
\end{tabular}

Сравнительный анализ заболеваемости детей и подростков прошлого века и настоящего времени позволил определить ведущие тенденции формирования хронической патологии у современных школьников:

- на сегодня ведущую позицию в структуре патологической пораженности занимают болезни костно-мышечной системы, которые составляют $31,68 \% \pm 0,46 \%$ от общего количества заболеваний;

- болезни глаза и придаточного аппарата занимают второе место, составляя $25,17 \% \pm$ $0,43 \%$ от общего количества заболеваний;

- на третье ранговое место поднимаются болезни эндокринной системы, которые составляют $11,32 \% \pm 0,32 \%$ от общего количества заболеваний;

- последующие четвёртое и пятое места занимают болезни системы кровообращения
$(10,05 \% \pm 0,30 \%)$ и расстройства питания и нарушений обмена веществ $(6,41 \% \pm 0,24 \%)$.

Исследованная за тридцатилетний срок патологическая пораженность школьников $6-16$ лет позволила выявить наивысший уровень заболеваемости именно в среднем школьном возрасте (рис. 2).

Поэтому в ходе дальнейших исследований было проведено прогнозирование динамики патологической пораженности детей среднего школьного возраста с целью планирования оздоровительных мероприятий для учеников с нарушениями в состоянии здоровья.

Проведенный ретроспективный анализ патологической пораженности школьников позволил разработать функциональные модели для прогноза отдельных классов заболеваний на краткосрочную перспективу. Полученные прогнозные значения параметров приведены в таблицах 2-3.

Таблица 1

Сравнительная характеристика патологической пораженности школьников 6 - 16 лет города Черкассы (интенсивные показатели \%о).

\begin{tabular}{|c|c|c|c|c|c|c|c|c|}
\hline \multirow{2}{*}{ Класс болезней } & \multicolumn{2}{|c|}{$1986-1988$ г, } & \multicolumn{2}{|c|}{$2013-2015$ г, } & \multirow{2}{*}{\begin{tabular}{c} 
Абсолютный \\
прирост \\
\cline { 2 - 7 }
\end{tabular}} & $P(\%)$ & $\begin{array}{c}\text { Темп } \\
\text { прироста } \\
(\%)\end{array}$ & $\begin{array}{c}\text { Темп } \\
\text { роста } \\
(\%)\end{array}$ \\
\hline $\begin{array}{c}\text { Системы } \\
\text { кровообращения }\end{array}$ & 21,13 & 0,53 & 74,12 & 0,98 & $<0,001$ & 52,99 & 250,78 & 350,78 \\
\hline $\begin{array}{c}\text { Глаза и его } \\
\text { придаточного } \\
\text { аппарата }\end{array}$ & 114,99 & 1,17 & 213,47 & 1,53 & $<0,001$ & 98,48 & 85,64 & 185,64 \\
\hline $\begin{array}{c}\text { Опорно- } \\
\text { двигательной } \\
\text { системы }\end{array}$ & 96,9 & 1,08 & 272,22 & 1,66 & $<0,001$ & 175,32 & 180,93 & 280,93 \\
\hline $\begin{array}{c}\text { Органов } \\
\text { пищеварение }\end{array}$ & 39,69 & 0,71 & 50,52 & 0,82 & $<0,001$ & 10,83 & 27,29 & 127,29 \\
\hline $\begin{array}{c}\text { Эндокринной } \\
\text { системы }\end{array}$ & 26,08 & 0,58 & 84,91 & 1,04 & $<0,001$ & 58,83 & 225,58 & 325,58 \\
\hline $\begin{array}{c}\text { Системы } \\
\text { дыхания }\end{array}$ & 30,75 & 0,63 & 56,51 & 0,86 & $<0,001$ & 25,76 & 83,77 & 183,77 \\
\hline $\begin{array}{c}\text { Нервной } \\
\text { системы }\end{array}$ & 18,07 & 0,49 & 24,58 & 0,58 & $<0,001$ & 6,51 & 36,03 & 136,03 \\
\hline $\begin{array}{c}\text { Мочеполовой } \\
\text { системы }\end{array}$ & 15,82 & 0,46 & 14,95 & 0,45 & $>0,05$ & $-0,87$ & $-5,50$ & 94,50 \\
\hline $\begin{array}{c}\text { Крови и } \\
\text { кроветворных } \\
\text { органов }\end{array}$ & 1,74 & 0,15 & 1,62 & 0,15 & $>0,05$ & $-0,12$ & $-6,90$ & 93,10 \\
\hline $\begin{array}{c}\text { Кожи и } \\
\text { подкожной } \\
\text { клетчатки }\end{array}$ & 6,57 & 0,30 & 3,75 & 0,23 & $<0,001$ & $-2,82$ & $-42,92$ & 57,08 \\
\hline
\end{tabular}




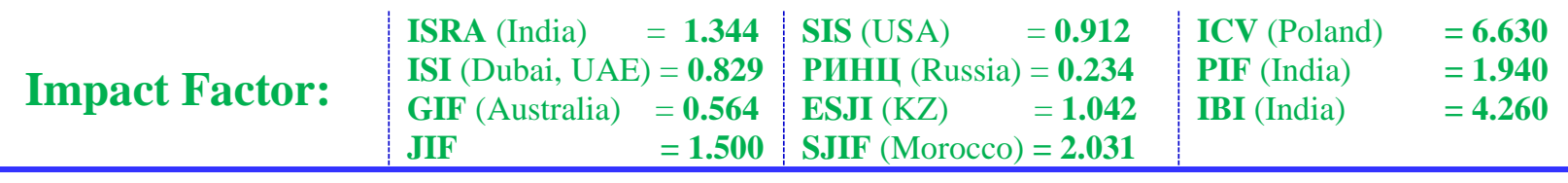

\begin{tabular}{|c|c|c|c|c|c|c|c|c|}
\hline Аллергии & 7,44 & 0,31 & 5,08 & 0,27 & $<0,001$ & $-2,36$ & $-31,72$ & 68,28 \\
\hline Другие болезни & 21,9 & 0,54 & 28,64 & 0,62 & $<0,001$ & 6,74 & 30,78 & 130,78 \\
\hline Вместе & 401,09 & 1,79 & 830,36 & 1,40 & $<0,001$ & 429,27 & 107,03 & 207,03 \\
\hline
\end{tabular}

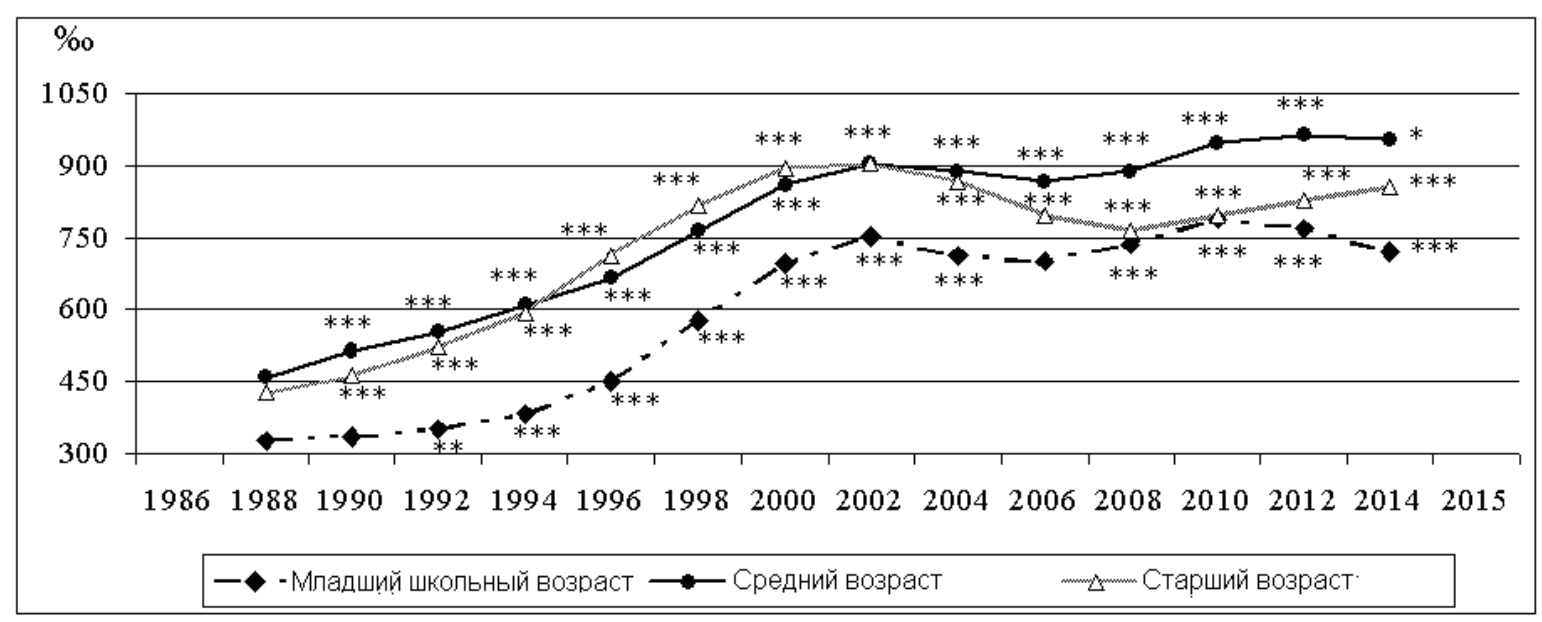

Рисунок 2 - Динамика патологической пораженности школьников разных возрастных категорій (сглаженные ряды данных в интенсивных показателях \%о).

Таблица 2

Параметры функциональной модели для прогноза динамики патологической пораженности детей среднего школьного возраста (модель линейного типа).

\begin{tabular}{|c|c|c|c|c|c|c|c|c|}
\hline \multirow{2}{*}{ Класс заболеваний } & \multicolumn{5}{|c|}{ Значения параметров } & \multicolumn{2}{c|}{ Прогноз (в \%о) } \\
\cline { 2 - 10 } & $a$ & $m_{\grave{a}}$ & $b$ & $m_{b}$ & $R$ & $p$ & 2016 г. & 2017 г. \\
\hline Системы кровоснабжения & $-5379,83$ & 673,76 & 2,72 & 0,33 & 0,91 & $<0,001$ & $\begin{array}{c}98,32 \\
\pm 12,72\end{array}$ & $\begin{array}{c}101,03 \\
\pm 13,34\end{array}$ \\
\hline $\begin{array}{c}\text { Глаза и его придаточного } \\
\text { аппарата }\end{array}$ & $-7093,58$ & 758,65 & 3,63 & 0,38 & 0,93 & $<0,001$ & $\begin{array}{c}220,43 \\
\pm 14,32\end{array}$ & $\begin{array}{c}224,06 \\
\pm 15,02\end{array}$ \\
\hline Костно-мышечной системы & $-15425,7$ & 1282,76 & 7,82 & 0,64 & 0,96 & $<0,001$ & $\begin{array}{c}338,12 \\
\pm 24,21\end{array}$ & $\begin{array}{c}345,93 \\
\pm 25,40\end{array}$ \\
\hline Нервной системы & $-1048,62$ & 267,66 & 0,54 & 0,13 & 0,73 & $<0,001$ & $\begin{array}{c}35,71 \\
\pm 5,05\end{array}$ & $\begin{array}{c}36,24 \\
\pm 5,30\end{array}$ \\
\hline Кожи и подкожной клетчатки & 259,73 & 60,61 & $-0,13$ & 0,03 & 0,75 & $<0,001$ & $\begin{array}{c}4,25 \\
\pm 1,14\end{array}$ & $\begin{array}{c}4,12 \\
\pm 1,20\end{array}$ \\
\hline Всего & $-38280,4$ & 4197,03 & 19,51 & 2,10 & 0,93 & $<0,001$ & $\begin{array}{c}1056,89 \\
\pm 79,22\end{array}$ & $\begin{array}{c}1076,40 \\
\pm 83,10\end{array}$ \\
\hline
\end{tabular}

Таблица 3

Параметры функциональной модели для прогноза динамики патологической пораженности детей среднего школьного возраста (модель полиномиального типа). 


\begin{tabular}{l|lr|ll|ll} 
& ISRA (India) & $=\mathbf{1 . 3 4 4}$ & SIS (USA) & $=\mathbf{0 . 9 1 2}$ & ICV (Poland) & $=\mathbf{6 . 6 3 0}$ \\
Impact Factor: & ISI (Dubai, UAE) $=\mathbf{0 . 8 2 9}$ & PVHL (Russia) & $=\mathbf{0 . 2 3 4}$ & PIF (India) & $=\mathbf{1 . 9 4 0}$ \\
& GIF (Australia) & $=\mathbf{0 . 5 6 4}$ & ESJI (KZ) & $=\mathbf{1 . 0 4 2}$ & IBI (India) & $=\mathbf{4 . 2 6 0}$ \\
& JIF & $\mathbf{1 . 5 0 0}$ & SJIF (Morocco) & $\mathbf{2 . 0 3 1}$ & & \\
\hline
\end{tabular}

\begin{tabular}{|c|c|c|c|c|c|c|}
\hline \multirow{2}{*}{ Класс заболеваний } & \multicolumn{3}{|c|}{ Значения параметров } & \multirow{2}{*}{$R$} & \multicolumn{2}{|c|}{ Прогноз (в \%о) } \\
\cline { 2 - 3 } \cline { 7 - 7 } & $a$ & $b$ & $c$ & & 2016 г. & 2017 г. \\
\hline Системы дыхания & -1135594 & 1133,70 & $-0,28$ & 0,92 & 54,27 & 46,93 \\
\hline Эндокринной системы & -540392 & 538,22 & $-0,13$ & 0,64 & 89,48 & 87,31 \\
\hline Органов пищеварения & $-434889,4$ & 433,32 & $-0,11$ & 0,78 & 74,41 & 72,48 \\
\hline Мочеполовой системы & 54896,57 & $-54,8814$ & 0,01 & 0,64 & 17,66 & 18,11 \\
\hline Другие заболевания & $-471834,23$ & 471,40 & $-0,12$ & 0,79 & 24,81 & 21,41 \\
\hline
\end{tabular}

Анализ функциональных моделей для прогноза отдельных классов заболеваний на краткосрочную перспективу позволил сформировать адаптивную модель заболеваемости школьников среднего школьного возраста, которая применена для моделирования и прогнозирования заболеваемости по отдельным классам заболеваний. Данная модель позволяет получить результаты, тренды прогнозов которых приближаются к показателям, рассчитанным ранее в линейной и полиномиальной функциональных моделях. Результаты расчетов основных параметров адаптивной модели и прогнозные значения приведены в таблице 4 .
Таким образом, полученные модели можно применять для прогнозирования динамики заболеваемости школьников среднего школьного возраста, для планирования профилактических и предупредительных комплексов мероприятий.

Для автоматизации процесса мониторинга и прогнозирования заболеваемости детей среднего школьного возраста было разработано программное обеспечение, структура которого представлена на рис. 3. Система моделирования заболеваемости школьников среднего школьного возраста состоит из модуля обработки входных данных, модуля формирования модели, модуля прогнозирования, модуля поддержки пользователя и интерфейса пользователя.

Таблица 4

Параметры адаптивной модели для прогноза динамики патологической пораженности учеников среднего школьного возраста.

\begin{tabular}{|c|c|c|c|c|c|c|}
\hline \multirow{2}{*}{ Класс заболеваний } & \multicolumn{3}{|c|}{ Значения параметров } & \multicolumn{3}{c|}{ Прогно (в \%о) } \\
\cline { 2 - 7 } & $a_{10}$ & $a_{20}$ & $r_{\varepsilon 0}$ & 2016 г. & 2017 г. & 2018 г. \\
\hline Системы кровоснабжения & 0,5826 & 0,4290 & 0,919 & $\begin{array}{c}85,853 \\
+5,145\end{array}$ & $\begin{array}{c}102,769 \\
\pm 2,646\end{array}$ & $\begin{array}{c}102,077 \\
+6,022\end{array}$ \\
\hline $\begin{array}{c}\text { Глаза и его придаточного } \\
\text { аппарата }\end{array}$ & 0,7386 & 0,2761 & 0,908 & $\begin{array}{c}230,493 \\
+4,287\end{array}$ & $\begin{array}{r}226,664 \\
\pm 2,273\end{array}$ & $\begin{array}{c}237,861 \\
+9,302\end{array}$ \\
\hline Костно-мышечной системы & 0,8814 & 0,1363 & 0,821 & $\begin{array}{c}320,858 \\
+7,861\end{array}$ & $\begin{array}{c}342,195 \\
+8,315\end{array}$ & $\begin{array}{c}365,829 \\
+9,872\end{array}$ \\
\hline Нервной системы & 0,8348 & 0,1819 & 0,921 & $\begin{array}{c}32,349 \\
+2,598\end{array}$ & $\begin{array}{c}36,033 \\
+2,243\end{array}$ & $\begin{array}{c}39,429 \\
+3,770\end{array}$ \\
\hline Кожи и подкожной клетчатки & 0,9321 & 0,0865 & 0,927 & $\begin{array}{c}4,55 \\
-0,236\end{array}$ & $\begin{array}{r}4,89 \\
-0,253\end{array}$ & $\begin{array}{c}4,77 \\
-0,341\end{array}$ \\
\hline Системы дыхания & 0,6174 & 0,3949 & 0,705 & $\begin{array}{c}58,550 \\
+4,361\end{array}$ & $\begin{array}{c}58,165 \\
+5,473\end{array}$ & $\begin{array}{c}42,484 \\
+4,643\end{array}$ \\
\hline Эндокринной системы & 0,9094 & 0,1087 & 0,750 & $\begin{array}{c}87,187 \\
+2,44\end{array}$ & $\begin{array}{c}86,758 \\
+9,691\end{array}$ & $\begin{array}{c}77,576 \\
+5,112\end{array}$ \\
\hline Органов пищеварения & 0,6977 & 0,3163 & 0,929 & $\begin{array}{c}72,244 \\
\pm 8,64\end{array}$ & $\begin{array}{c}55,35 \\
+5,70\end{array}$ & $\begin{array}{c}55,82 \\
+7,03\end{array}$ \\
\hline Мочеполовой системы & 0,9781 & 0,0415 & 0,932 & $\begin{array}{c}16,507 \\
+0,952\end{array}$ & $\begin{array}{c}18,673 \\
+1,893\end{array}$ & $\begin{array}{c}18,390 \\
+2,252\end{array}$ \\
\hline Всего & 0,8297 & 0,1869 & 0,930 & $\begin{array}{c}973,333 \\
+38,462\end{array}$ & $\begin{array}{c}1035,853 \\
+58,525\end{array}$ & $\begin{array}{c}1061,102 \\
+68,572\end{array}$ \\
\hline
\end{tabular}

ISPC Results and Perspectives, 


\begin{tabular}{l|lrl|l|ll} 
& ISRA (India) & $=\mathbf{1 . 3 4 4}$ & SIS (USA) & $=\mathbf{0 . 9 1 2}$ & ICV (Poland) & $=\mathbf{6 . 6 3 0}$ \\
Impact Factor: & ISI (Dubai, UAE) $=\mathbf{0 . 8 2 9}$ & PUHU (Russia) $=\mathbf{0 . 2 3 4}$ & PIF (India) & $=\mathbf{1 . 9 4 0}$ \\
& GIF (Australia) & $\mathbf{0 . 5 6 4}$ & ESJI (KZ) & $=\mathbf{1 . 0 4 2}$ & IBI (India) & $\mathbf{4 . 2 6 0}$
\end{tabular}

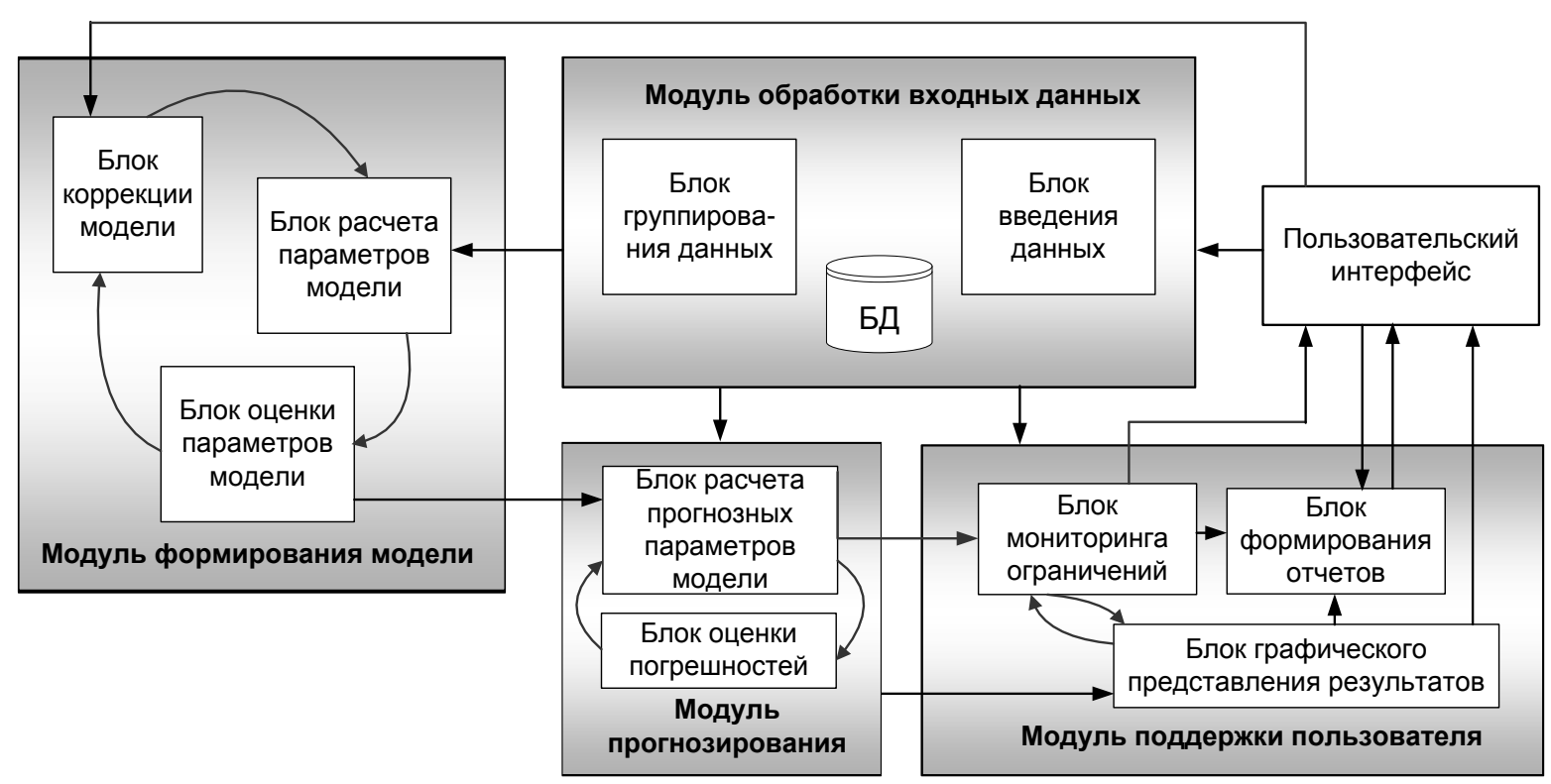

Рисунок 3 - Структура системы моделирования заболеваемости детей среднего школьного возраста.

После группирования входных данных (в одноименном блоке) происходит расчет параметров и формирование модели заболеваемости школьников среднего школьного возраста, которая в дальнейшем передается для прогнозирования (в модуль прогнозирования). Такая передача модели из модуля формирования модели возможна только после оценки качества модели на числовых рядах входных данных, которые указаны пользователем, как контрольные. Возможно также участие пользователя в формировании модели, что обеспечивается функциями выбора базовой модели и наборов начальных значений параметров.

В модуле прогнозирования проводиться непосредственный расчет прогнозных значений и оценок их отклонений. В модуле поддержки пользователя проводиться визуализация существующих и прогнозных значений показателей заболеваемости школьников. В этом модуле реализованы функции по отслеживанию и контролю динамики заболеваемости по различным видам заболеваний и в целом, а также проводиться формирование отчетов.

\section{5. Обсуждение результатов исследований состояния здоровья школьников}

Проведенный, за период с 1986 по 2015 год, ретроспективный анализ состояния здоровья школьников 6 - 16 лет города Черкассы выявил неблагоприятную хронодинамику, которой присущ рост уровня патологической пораженности учеников, этот уровень повысился более чем вдвое с 385,91\% \pm 4,07\%о в 1986 году до $810,39 \%$ \% $2,50 \%$ в 2015 году $(p<0,001)$ (рис. 1). Следовательно, ухудшение состояния здоровья детей и подростков центрального региона Украины за исследуемые 30 лет является доказанным фактом.

На следующем этапе исследования с помощью экстенсивных показателей изучалась структура патологической пораженности детей и подростков 6 - 16 лет. В соответствии с полученными данными, в начале наблюдения $27,19 \% \pm 0,37 \%$ школьников были поражены болезнями глаза и придаточного аппарата. Четверть учеников имели болезни и функциональные нарушения костно-мышечной системы $(24,63 \% \pm 0,36 \%$ от общего количества заболеваний). На третьем по распространенности месте были болезни органов пищеварения $(12,65 \% \pm 0,28 \%$ от общего количества заболеваний). Четвертое же место разделяли болезни системы дыхания $(7,43 \% \pm 0,22 \%$ от общего количества заболеваний) и болезни эндокринной системы, расстройства питания и нарушения обмена веществ $(6,11 \% \pm 0,20 \%$ от общего количества заболеваний) (табл. 1).

В последние годы наблюдения отмечается перераспределение наиболее распространенных заболеваний. Так, в результате исследований выяснено, что в 2015 году в структуре патологической пораженности преимущество принадлежит болезням и функциональным нарушениям костно-мышечной системы, которые имеет треть современных школьников $(31,68 \% \pm 0,46 \%)$. С первого на второе ранговое место опускаются болезни глаза и его придаточного аппарата $(25,17 \% \pm 0,43 \%$ от общего количества заболеваний). На третье место поднимаются болезни эндокринной системы, расстройства питания и нарушения обмена 
веществ $(11,32 \% \pm 0,32 \%$ от общего количества заболеваний) вместе с болезнями системы кровообращения $(10,05 \% \pm 0,30 \%$ от общего количества заболеваний). Четвертое же занимают болезни органов пищеварения $(6,41 \% \pm 0,24 \%$ от общего количества заболеваний) (табл. 1). Таким образом, анализ структуры заболеваемости школьников 6 - 16 лет указал на количественные и качественные изменения ведущей патологии, которые произошли за последние тридцать лет.

На следующем этапе исследований при распределении детей по возрасту было установлено, что на протяжении почти всего исследуемого периода, именно в среднем школьном возрасте отмечается явление активной хронизации болезней (рис. 2), а следовательно школьники этой возрастной группы являются наиболее уязвимым звеном и поэтому нуждаются в особенном внимании.

В последующем, на основе рассчитанных коэффициентов заболеваемости учеников среднего школьного возраста, нами строились динамические ряды данных по отдельными нозологиям, которые позволили проследить за характером и динамическими изменениями этих заболеваний на протяжении последних тридцати лет.

Полученные при проведении исследований функциональные и адаптивная модели позволяют с достаточно высоким уровнем вероятности прогнозов получить показатели динамики развития заболеваний по выделенным классам. Это результаты предусматривают негативный характер динамики патологической пораженности детей среднего школьного возраста, которая в 2016 - 2018 годах может увеличиться до показателей $\quad 1056,89 \%$ о $\pm 79,22 \%$

$1076,40 \% \pm 83,10 \%$ o $\quad(R=0,93 ; \quad p<0,001)$ (функциональная модель), 1035,85 \%o + 58,53\%о $1061,10 \%+68,57 \%$ (адаптивная модель), в большей степени за счет возрастания удельного веса заболеваний и функциональных нарушений костно-мышечной системы до 338,12 \% $\pm 24,21 \%$

$345,93 \%$ \% $\pm 25,40 \%$ o $\quad(R=0,96 ; \quad p<0,001)$ (функциональная модель), 342,2 \%о +8,32\%о $365,83 \%$ + 9,87\% (адаптивная модель), глаза и его придаточного аппарата до $220,43 \%$ o $\pm 14,32 \%$ - $\quad 224,06 \% \pm 15,02 \%$ o $\quad(R=0,93 ; \quad p<0,001)$ (функциональная модель), 230,49\%о + 4,29\% $237,86 \%$ + 9,3\%о (адаптивная модель), болезней системы кровоснабжения до 98,32 \% $\pm 12,72 \%$ $101,03 \%$ \% $13,34 \%$ o $\quad(R=0,91 ; \quad p<0,001)$ (функциональная модель), 102,077 \%о + 6,02\% $102,77 \%$ + 2,65 \%о (адаптивная модель).

Однако, приостановка роста уровня патологической пораженности в среднем школьном возрасте будет происходить преимущественно за счет снижения удельного веса заболеваний органов дыхания до 54,27 \%о46,93\%о (функциональная модель), 58,17 \%о $42,48 \%$ (адаптивная модель), заболеваний органов пищеварения до 74,41 \%o-72,48 \%о (функциональная модель), 72,24 \%о - 55,82 \%о (адаптивная модель), и заболеваний эндокринной системы до 89,48 \%о-87,31 \%о (функциональная модель), 86,75 \% - 77,58 \%о (адаптивная модель).

Опыт моделирования дальнейшего уровня заболеваемости в ряде стран $[15,16]$ доказывает его целесообразность при планировании профилактических мероприятий, которые направлены на уменьшение заболеваемости населения страны. Полученные в процессе проведенного исследования функциональные и адаптивная модели позволяют предусмотреть характер динамики патологической пораженности школьников и в результате спланировать оздоровительно-профилактические мероприятия, направленные на снижение ведущей патологии в школьном возрасте.

\section{Выводы}

Важной задачей современной медицины является обоснованное планирование и проведение оздоровительных мероприятий, направленных на профилактику наиболее распространенных патологий. Основным потенциалом здоровья населения являются дети школьного возраста, заболевания которых связаны с негативными экологическими, экономическими и социальными факторами. В связи с увеличением уровня хронической патологии среди современных детей школьного возраста в Украине, необходимо расширять существующие научные исследования для планирования оздоровительных программ, которые направлены на профилактику заболеваний школьников с нарушениями в состоянии здоровья. Прогнозируемый дальнейший рост уровня патологической пораженности детей школьного возраста определяет необходимость применения дополнительных оздоровительно-профилактических мероприятий, направленных на приостановку неблагоприятных тенденций в состоянии здоровья современных школьников.

Сформированные модели и полученные результаты данного исследования позволяют проводить планирование и проведение оздоровительных мероприятий, а также могут использоваться специалистами лечебнопрофилактических учреждений территориальных детских поликлиник и персоналом общеобразовательных учебных заведений для целенаправленного планирования различных видов помощи для детей с нарушениями в состоянии здоровья. 


\begin{tabular}{l|lrl|l|ll} 
& ISRA (India) & $=\mathbf{1 . 3 4 4}$ & SIS (USA) & $=\mathbf{0 . 9 1 2}$ & ICV (Poland) & $=\mathbf{6 . 6 3 0}$ \\
Impact Factor: & ISI (Dubai, UAE) $=\mathbf{0 . 8 2 9}$ & PUHU (Russia) $=\mathbf{0 . 2 3 4}$ & PIF (India) & $=\mathbf{1 . 9 4 0}$ \\
& GIF (Australia) & $\mathbf{0 . 5 6 4}$ & ESJI (KZ) & $=\mathbf{1 . 0 4 2}$ & IBI (India) & $\mathbf{4 . 2 6 0}$
\end{tabular}

\section{References:}

1. Nyagu AI (2011) Health of survivors in Ukraine in 25-years dynamics after the Chernobyl catastrophe, Congress the Chernobyl Catastrophe: Taking Stock of 25 Years of Ecological and Health Damages. Berlin. Available:

http://www.chernobylcongress.org/fileadmin/use r_upload/pdfs/nyagu.pdf

(Accessed: 12.06.2016).

2. Foley TP Jr, Límanová Z, Potluková E (2015) Medical consequences of Chernobyl with focus on the endocrine system: Part 1. Cas Lek Cesk, No.154(5), pp.227-31.

3. Foley TP Jr, Límanová Z, Potluková E (2015) Medical Consequences of Chernobyl with Focus on the Endocrine System - Part 2. Cas Lek Cesk, No.154(6), pp.287-91.

4. Cardis E, Hatch M (2011) The Chernobyl accident-an epidemiological perspective. Clin Oncol (R Coll Radiol), No.23(4), pp.251-60. DOI: 10.1016/j.clon.2011.01.510

5. Tukiendorf A, Miszczyk L, McEwan P (2010) Recent Epidemiological Results of Thyroid Cancer in the Most Radiated Territory in Poland Cent Eur J Public Health, No.18(3), pp.157-160.

6. Boyarchuk D, Betliy O, Orlova I (2011) Public Expenditures on Education and Health in Ukraine Before and During the Global Crisis. CASE Network Reports, Warsaw, No.104, pp.72.

7. Piekkala A (2012) Challenges of Public Heals Education in the former Soviet Union: Example of Ukraine. Tobacco Control and Public Heals in Eastern Europe, Vol. 2, No. 2, pp.67-76.

8. Lazarus JV, Balabanova D, McKee M (2012) Making the First Global Society for Health Systems Research Truly Global. Cent Eur J Public Health, No.20(4), pp.299-300.

9. McKee M, Stuckler D, Basu S (2012) Where there is no health research: what can be done to fill the global gaps in health research? PLoS medicine, No.9(4), e1001209.
10. (2016) Postanova KMU za № 1318 vid 08.12.09 r. pro zatverdgennja "Porjadku zdiysnrnnja medyshnogo obslugovuvannja ushniv zagalnoosvitnih navzalnyh zakladiv". Available: http://zakon.rada.gov.ua/cgi-

bin/laws/main.cgi?nreg=1318-2009-\%EF

(Accessed: 17.06.2016).

11. (2010) Nakaz MOZ Ukrainy za № $682 \operatorname{vod}$ 16.08.2010. "Pro udoskonalennja medyshnogo obslugovuvannja ushniv zagalnoosvitnih navzalnyh zakladiv". Available: http://zakon.rada.gov.ua/cgibin/laws/main.cgi?nreg=z0794-10 (Accessed: 17.06.2016).

12. Petrov AI (2003) Statisticheskaja teorija radiotehnisheskih system: usheb. posob. M., Izdvo Radiotechnika, p. 400. ISBN 5-93108-047-3.

13. Sage AP, Melse JL (1972) Estimation Theory with Application to Communication and Control. N.-Y. McGraw-Hill, pp.496.

14. Kashyap R, Rao A (1976) Dynamic stochastic models from empirical data. Academic Press, New York, pp.344.

15. Webber L, Halicka E, Marsh T, Rtveladze K, McPherson K, Brown M (2014) Projected Incidence of Overweight and Obesity and Related Disease Incidence Across Poland. Cent Eur J Public Health, No.22(1), pp.17-23.

16. Webber L, Divajeva D, Marsh T, McPherson K, et al. (2014) The future burden of obesity-related diseases in the 53 WHO European-Region countries and the impact of effective interventions: a modelling study. BMJ Open, No.4, e004787. doi: 10.1136/bmjopen-2014004787. Available: https://www.researchgate.net/ publication/264246821_The_future_burden_of_ obesity-related_diseases_in_the_53_WHO_Euro pean-Region_countries_and_the_impact_of_effe ctive_interventions_A_modelling_study (Accessed 7.05.2016). 